\title{
Cosmography of OB stars in the solar neighbourhood ${ }^{\star}$
}

\author{
H. Bouy ${ }^{1}$ and J. Alves ${ }^{2}$ \\ 1 Centro de Astrobiología, INTA-CSIC, Depto Astrofísica, ESAC Campus, PO Box 78, 28691 Villanueva de la Cañada, \\ Madrid, Spain \\ e-mail: hbouy@cab.inta-csic.es \\ 2 Department of Astrophysics, University of Vienna, Türkenschanzstrasse 17, 1180 Vienna, Austria
}

Received 25 July 2015 / Accepted 6 October 2015

\begin{abstract}
We construct a 3D map of the spatial density of OB stars within $500 \mathrm{pc}$ from the Sun using the HiPPARcos catalogue and find three large-scale stream-like structures that allow a new view on the solar neighbourhood. The spatial coherence of these blue streams and the monotonic age sequence over hundreds of parsecs suggest that they are made of young stars, similar to the young streams that are conspicuous in nearby spiral galaxies. The three streams are 1) the Scorpius to Canis Majoris stream, covering $350 \mathrm{pc}$ and $65 \mathrm{Myr}$ of star formation history; 2) the Vela stream, encompassing at least $150 \mathrm{pc}$ and $25 \mathrm{Myr}$ of star formation history; and 3) the Orion stream, including not only the well-known Orion OB1abcd associations, but also a large previously unreported foreground stellar group lying only $200 \mathrm{pc}$ from the Sun. The map also reveals a remarkable and previously unknown nearby OB association, between the Orion stream and the Taurus molecular clouds, which might be responsible for the observed structure and star formation activity in this cloud complex. This new association also appears to be the birthplace of Betelgeuse, as indicated by the proximity and velocity of the red giant. If this is confirmed, it would solve the long-standing puzzle of the origin of Betelgeuse. The well-known nearby star-forming low-mass clouds, including the nearby $\mathrm{T}$ and $\mathrm{R}$ associations Lupus, Cha, Oph, CrA, Taurus, Vela R1, and various low-mass cometary clouds in Vela and Orion, appear in this new view of the local neighbourhood to be secondary star formation episodes that most likely were triggered by the feedback from the massive stars in the streams. We also recover well-known star clusters of various ages that are currently cruising through the solar neighbourhood. Finally, we find no evidence of an elliptical structure such as the Gould belt, a structure we suggest is a $2 \mathrm{D}$ projection effect, and not a physical ring.
\end{abstract}

Key words. stars: massive - solar neighborhood - open clusters and associations: general

\section{Introduction}

Because of their relatively short lifetimes, massive $\mathrm{O}$ and $\mathrm{B}$ stars are a good tracer of recent star formation in the Milky Way and nearby galaxies. It was noted very early on that $\mathrm{O}$ and B stars are not distributed randomly on the sky (Herschel 1847; Gould 1879). Following the early canonical work of Eddington (1914), Kapteyn (1914), Charlier (1926), and Pannekoek (1929), Blaauw (1964) analysed the spatial and velocity distributions of OB stars in the solar neighbourhood to identify and study the content of nearby stellar groups. At the end of the last century, the accuracy and whole-sky coverage of the Hipparcos mission led to a major improvement in the definition and characterization of nearby $\mathrm{OB}$ associations and clusters, which profoundly changed our knowledge and understanding of the solar vicinity (e.g. Figueras et al. 1997; de Zeeuw et al. 1999; Platais et al. 1998; Subramaniam \& Bhatt 2000; Hoogerwerf 2000; de Bruijne 2000; Branham 2002; Elias et al. 2006a,b).

There are two predominant methods for identifying stellar groups in the literature. The first consists of searching for concentrations in $2 \mathrm{D}$ projections of the position and/or velocity spaces. Starting with John Herschel and Benjamin Gould's original observations, OB stars were grouped in a "belt" on the projected sky; this is known today as the Gould belt. As precise photometric or parallactic distance measurements were accumulating, the searches were performed in all possible 2D projections of their $X, Y, Z$ Cartesian galactic coordinates

\footnotetext{
$\star$ Table 3 is available in elctronic form at http://www . aanda.org
}

(e.g. Charlier 1926; Stothers \& Frogel 1974; Westin 1985; Elias et al. 2006b, and references therein). At the beginning of the twentieth century, the advent of precise proper motion measurements (and to a lesser extent, radial velocities) opened the search space to velocities. When the mean proper motion of a group is sufficiently high to distinguish it from the quasi-random velocity distribution of disk stars, its co-moving members are identified in $2 \mathrm{D}$ vector-point diagrams. Whenever radial velocities were also available, the search for co-moving stars was often made in 2D projections of their Cartesian galactic velocities $(U$ vs. $V, V$ vs. $W$, and $U$ vs. $W$, e.g. Eggen 1984 ; Torres et al. 2006; Elias et al. 2006a, and references therein). But 2D projections are incapable of describing all the features of a 3D space, even if multiple projections are considered simultaneously. Important structures can be lost, hidden in the projection, while artificial structures can appear.

The second main procedure used to identify stellar groups relies on the convergent point method originally developed by Charlier that continued to be refined over the years (e.g. de Bruijne 1999; Galli et al. 2012). Because of perspective effects, groups of co-moving stars tend to move towards a common convergence point on the sky, while unrelated stars will move towards random directions. If a co-moving group exists, the paths defined by its members will intersect at the convergence point and the distribution of intersection points will be denser in that direction. The convergent point method has been widely and successfully used and led to the discovery or confirmation of most well-known $\mathrm{OB}$ associations and clusters listed in the extensive 
inventory of de Zeeuw et al. (1999). But it suffers from a major bias towards groups with high tangential motion. When the motion of a stellar group is mostly radial, the convergent point coincides with the projected geometric centre of the group. The discovery of such a group is often impeded because the typical relative error on the proper motion of current surveys is usually too large. This bias will in particular affect the identification of stellar groups at large heliocentric distances or located towards the solar apex or antapex. We show in the course of the present study the surprising consequences in the Orion and Vela starforming complexes.

Finally, both methods can be affected by the presence of companions. The orbital motion of long-period binaries can add a significant non-linear component to the apparent motion and alter the proper motion measurements. This effect is particularly relevant for OB stars because of their high multiplicity rate. It did not affect the discovery of large conspicuous groups of OB stars whose co-motion will statistically dominate (de Zeeuw et al. 1999). But it might prevent the discovery of smaller and sparser groups of OB stars by shuffling the velocity measurements of a significant fraction of their members and diffusing the group coherence in the convergent point diagram. Therefore velocities should be used with caution when blindly searching for small groups of OB stars, and in particular in the subsequent assignment of membership probabilities to individual stars.

In the present study, we use the HiPParcos catalogue to revisit the cosmography of OB stars in the solar neighbourhood. Because of the drawbacks mentioned above, we focus on the 3D spatial distribution using modern full 3D data analysis and interactive visualization techniques instead of $2 \mathrm{D}$ projections, and refrain from using velocities as a discovery criterion for stellar groups.

\section{Building the sample of nearby $O B$ stars in the Hipparcos catalogue}

We selected all the stars belonging to the $\mathrm{O}$ and $\mathrm{B}$ spectral classes among the 117955 sources of the van Leeuwen (2007) catalogue. The spectral types listed in the HiPPARcos catalogue originate from very diverse sources, and their precision and reliability are difficult to assess (ESA 1997). A few sources at the late-type end might be mis-classified, but the inclusion of a few earlyA stars or the exclusion of a few late-B stars is not expected to have any significant influence on our analysis of galactic structures that are made of massive stars.

The Hipparcos Input Catalogue is an amalgamation of two main components. The survey component was defined as a magnitude-limited sample as complete as possible up to $V \sim$ 8 mag and covering uniformly the celestial sphere. The second component included selected targets from more than 200 scientific proposals and is neither complete nor uniform over the entire sky. The next step in building a complete and uniform sample of nearby OB stars therefore consisted of selecting only survey stars.

Finally, all survey OB stars falling within a box of galactic rectangular coordinates defined by

$$
\begin{aligned}
& --500<X<500 \mathrm{pc} \\
& --500<Y<500 \mathrm{pc} \\
& --200<Z<200 \mathrm{pc}
\end{aligned}
$$

were selected. The limits in $X$ and $Y$ were chosen to amply encompass the volume set by the magnitude limit of the survey for
OB stars $(400 \sim 500 \mathrm{pc})$ as well as most well-known nearby clusters and associations. The range in $Z$ approximately corresponds to the thickness of the thin galactic stellar disk and also includes most well-known clusters and associations of the solar neighbourhood. The transformation from parallax to distance required to compute the galactic Cartesian coordinates must be performed with caution. The usual formula $d=1 / \varpi$ gives a biased estimator of the distance, with a skewed probability distribution of $d$ depending on the relative error on the parallax $\epsilon=\sigma_{\varpi} / \varpi$. A common practice for minimizing this bias consists of limiting the sample to stars with a small relative error on the parallax, and we selected sources satisfying $\epsilon \leq 0.25$.

The final sample is made of ten $\mathrm{O}$ and $3523 \mathrm{~B}$ stars. A number of biases related to the physical properties of the sample arise and affect the study. Extinction will in some case severely alter the completeness of the sample. Undetected multiplicity, which can be frequent among OB stars, is also a source of errors on the parallax and complicates certain aspects of the analysis. But with these limitations on the completeness of the sample in mind, the spatial distribution of OB stars in Hipparcos can be used to probe large-scale ( $>10 \mathrm{pc}$ ) local galactic stellar structures within $500 \mathrm{pc}$.

\section{Cosmography of $\mathrm{OB}$ stars in the solar neighbourhood}

We chose to study the spatial distribution of OB stars in galactic rectangular $X Y Z$ coordinates using a 3D kernel density estimator (KDE). The KDE offers several advantages. First, it delivers a non-parametric description of the spatial density with no assumption about the spatial structures present in the sample. Second, it transforms a discrete collection of scattered individual measurements into a continuous distribution of density ideally suited to reveal stellar groups. Third, it smooths out the contribution of each observed data point over a local neighbourhood of that data point and should therefore deliver a more robust estimate of the average distances to the groups of OB stars (over-densities) in which this study is interested. While individual HipParcos parallaxes become increasingly unreliable beyond $\sim 100$ pc, relatively accurate mean distances have indeed been measured for stellar groups as far as $650 \mathrm{pc}$ (e.g. de Zeeuw et al. 1999).

We chose a Gaussian kernel and an isotropic bandwidth of $12 \mathrm{pc}$. This value was chosen heuristically as a good compromise between over- and under-smoothing physical density enhancements among random density fluctuations. Smaller kernels gave noisy distributions that revealed every single star as a density enhancement, while broader kernels washed out important wellknown stellar groups. The main structures identified below are at least an order of magnitude larger than the chosen bandwidth, which ensures that the choice of bandwidth does not dominate the size scale of the structures of interest.

Some observational biases affect the sample and the KDE analysis.

1. The Malmquist bias introduced by the magnitude-limited nature of the Hipparcos survey. Assuming that there is no extinction, the $V \sim 8 \mathrm{mag}$ approximate limit of the survey corresponds to an horizon of approximately $450 \mathrm{pc}$ for a B9V star. This number can probably be used as a very rough approximation of the completeness limit and suggests that the Malmquist bias probably does not affect our analysis significantly. 


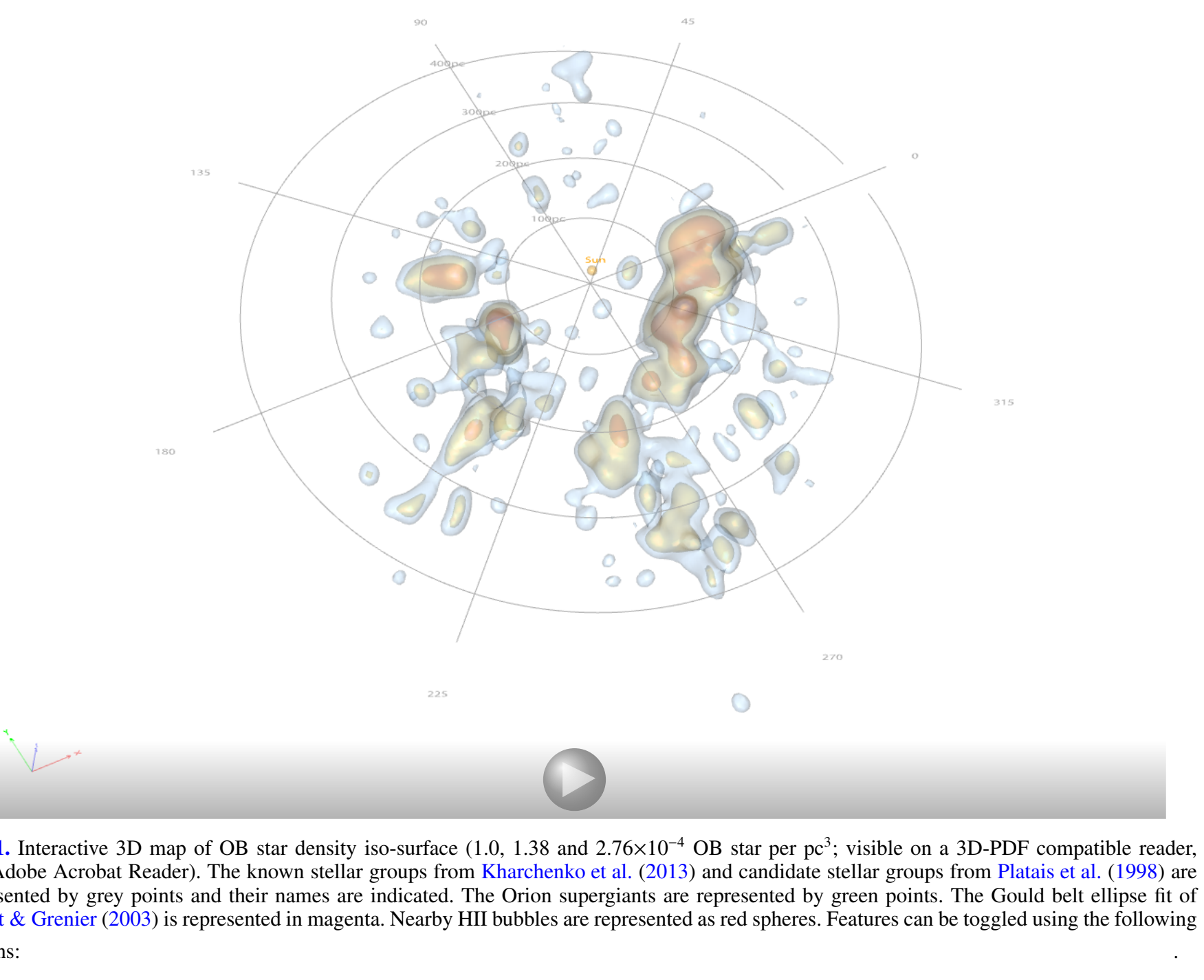

Fig. 1. Interactive 3D map of OB star density iso-surface $\left(1.0,1.38\right.$ and $2.76 \times 10^{-4} \mathrm{OB}$ star per pc $\mathrm{pc}^{3}$; visible on a 3D-PDF compatible reader, like Adobe Acrobat Reader). The known stellar groups from Kharchenko et al. (2013) and candidate stellar groups from Platais et al. (1998) are represented by grey points and their names are indicated. The Orion supergiants are represented by green points. The Gould belt ellipse fit of Perrot \& Grenier (2003) is represented in magenta. Nearby HII bubbles are represented as red spheres. Features can be toggled using the following

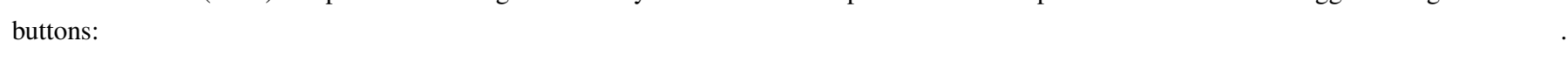

2. The selection bias due to the restriction in parallax, described in Lutz \& Kelker (1973). This bias must affect the KDE analysis. We ran some simple Monte Carlo simulations assuming a cluster with 1000 stars, a distribution of parallax errors similar to that of the HiPPARcos catalogue, cluster sizes in the range between $10-50 \mathrm{pc}$, and distances between 100-00 pc. They showed that the distance to the peak of the KDE can be underestimated by $2 \%$ to $7 \%$.

3. The truncation in relative parallax error leads to a preference for the brightest (smaller errors) and/or nearest (high parallax) stars (Brown et al. 1997). At the same time, the truncation limits the bias in the distance to $10 \%$ on average (overestimate) with a variance of $50 \%$, as described in Bailer-Jones (2015).

The interplay between these biases and their effect on the spatial distribution of OB stars in the solar neighbourhood are difficult to estimate. An exhaustive investigation of their effects is beyond the scope of the present study, and the expected levels of error are not expected to compromise the existence and basic properties of the large structures revealed in our analysis.

Appropriate visualization is critical to enhance the comprehension of spatial relationships in multivariate data and explore, analyse, and interpret the results obtained (Goodman 2012; Barnes et al. 2013). Interactivity is particularly important to avoid the problems and limitations of orthographic or isometric projections when exploring the data. Projecting a 3D space into spaces of lower dimension can indeed easily result in situations where distortions are strong, distances, depth, and altitude are difficult to gauge, and the interpretation will often depend strongly on the subjective choice of a viewpoint. In Fig. 1 we present the 3D density map of $\mathrm{OB}$ stars in the solar neighbourhood. The figure was constructed using $R$ and its $r g l$ package ( $\mathrm{R}$ Core Team 2015; Adler et al. 2015) and displays the result of the 3D KDE. A 3D-PDF compatible reader (like Adobe Acrobat Reader) or WebGL compatible web browser are required for the visualization. Three levels of isodensity are represented, for $1.00,1.38$, and $2.76 \times 10^{-4} \mathrm{OB}$ star $\mathrm{pc}^{-3}$, corresponding to $0.75,1$, and $2 \mathrm{OB}$ stars in a $12 \mathrm{pc}$ radius sphere.

A number of structures are clearly apparent, most of which can be recognized. Well-known clusters, including $\alpha$-Per (Per OB3), the Pleiades, Messier 7, IC 2602, IC 2391, NGC 2451A, Collinder 135, $\gamma$ Velorum, and NGC 2516 clearly appear as density enhancements. The Sco OB2, Vela OB2, and Trumpler $10 \mathrm{OB}$ associations are also discernible in the 3D KDE map (Fig. 1). 
It must be realized that the present KDE analysis is inevitably biased because the single-kernel bandwidth introduces a bias towards certain spatial scales. For example, the loose Lac OB1, Cep OB6, and Cas-Tau OB associations are missed in the 3D map even though they lie within the 500 pc limit of our analysis, mostly because they are too sparse to be revealed with the chosen kernel bandwidth and density levels. The same applies to the two main clusters of Per OB2: IC 348 contains only two B stars and is just visible at the lowest density level represented here, while NGC 1333, also including two B stars, is still deeply embedded in its parental molecular cloud (e.g. Lada et al. 1996). As already mentioned above, extinction indeed severely affects the completeness of the HipParcos survey. Even though Orion OB1 is known to harbour the most massive nearby clusters and the densest concentration of OB stars, the relatively large distance (between 380 and $420 \mathrm{pc}$ ), aggravated in part by dust extinction, results in an artificially low density, and the well-known groups are invisible at the iso-density levels represented in Fig. 1. By studying massive $\mathrm{O}$ and $\mathrm{B}$ stars alone, our study is also biased towards young ( $\$ 100 \mathrm{Myr})$ stellar groups because older associations or clusters have lost their most massive members either because of evolutionary or dynamical processes. Finally, lowmass $T$ and $R$ associations are naturally missed since they do not contain any massive stars.

In the near future, Gaia is hoped to provide the accuracy and sensitivity required to look deeper into the clouds, include stars of later spectral types, perform multi-resolution analysis, and reveal these missed associations and clusters. In the following, we concentrate on the densest groups of OB stars as probed by our KDE analysis, keeping in mind that the present census is not complete and biased towards young ( $\lesssim 100 \mathrm{Myr}$ ) dense $\left(\gtrsim 10^{-4} \mathrm{OB}\right.$ star $\left.\mathrm{pc}^{-3}\right)$ stellar groups with little line-of-sight extinction.

Three major structures associated with well-known starforming complexes and similar to the "streams of helium stars" described by Eddington (1914) and Kapteyn (1914) are revealed in Fig. 1. Following Kapteyn, we should name the structures "streams", but to avoid confusion with the current usage of the word "stream" in the literature (associated with stellar structures created by disrupted dwarf galaxies or clusters orbiting larger galaxies) we instead use "blue streams". Qualifying the word "streams" with the colour blue seems appropriate because these structures are made of young stellar populations containing blue OB stars. In the following section, we review the three newly identified blue streams sequentially.

\subsection{Scorpius-Canis Majoris blue stream}

Building on the early studies of Plummer (1913), Kapteyn (1914), Rasmuson (1921), and Charlier (1926), the seminal work of Blaauw (1946) on OB associations identified the Sco OB2 (Sco-Cen) association as the nearest stellar association to the Sun. Following Kapteyn's original description, Blaauw (1964) divided it into three sub-groups: Upper Sco (USco), Upper Centaurus-Lupus (UCL), and Lower Cen-Crux (LCC). As expected, all three components of the Sco OB2 complex appear clearly visible in the 3D density map. But Fig. 1 also shows that the complex extends well beyond LCC and up to at least $l \sim 240^{\circ}$ in the Canis Majoris constellation, forming a $350 \mathrm{pc}$ long blue stream. Over-plotting the list of nearby clusters in the corresponding area (from Kharchenko et al. 2013) reveals that most over-densities beyond LCC longitude are associated with well-known clusters: IC 2602, IC 2391, a Car, NGC 2451A,
Table 1. Ages assumed for the Sco-CMa clusters and associations.

\begin{tabular}{lcc}
\hline \hline Group & Age & Ref. \\
\hline USco & $4-11$ & $(1),(2)$ \\
UCL & 16 & $(1)$ \\
LCC & 17 & $(1)$ \\
IC 2602 & $46 \pm 6$ & $(3)$ \\
IC 2391 & $50 \pm 5$ & $(4)$ \\
a Car & 60 & $(5)$ \\
Col135 & 40 & $(6),(8)$ \\
NGC 2451A & $65 \pm 15$ & $(7)$ \\
\hline
\end{tabular}

References. (1) Pecaut et al. (2012); (2) Herczeg \& Hillenbrand (2015); (3) Dobbie et al. (2010); (4) Barrado y Navascués et al. (2004); (5) Platais et al. (1998); (6) Claria \& Kepler (1980); (7) Hünsch et al. (2003); (8) Rosario et al. (2013).

Collinder 135, and the cluster candidate reported by Platais et al. (1998) around HIP 45189. The existence of an old association including Collinder 135 and NGC 2451A has previously been suggested by Williams (1967), who called it the Pup-Can association. Our analysis corroborates the existence of a large complex between Puppis and Canis Majoris including Collinder 135 and NGC 2451A, but suggests that it is in fact the continuation of the Sco OB2 complex. We note that the tail of the stream does not correspond to any of the Puppis and CMa OB associations and complexes reported in Kaltcheva \& Hilditch (2000), which were later re-discovered by Caballero \& Dinis (2008) and are all located farther away.

Even more striking than the spatial coherence and continuity over almost $350 \mathrm{pc}$, the OB associations and clusters inside the stream display remarkable properties strongly suggesting that they must be genetically linked. Table 1 and Figs. 2 to 4 show that the associations and clusters are ordered by increasing age, starting from the young ( 4 11 Myr) USco, and sequentially passing through the 15-20 Myr UCL and LCC, the 30-50 Myr a Car, IC 2602, IC 2391, and Collinder 135 open clusters, to finally reach the 65 Myr NGC 2451A open cluster. Figure 3 shows that within the large uncertainties, the ages of the stellar groups belonging to the stream are correlated to their relative distances, suggesting a continuous propagation of the star formation along the stream. In total, the Sco-CMa stream covers a period of star formation of at least $65 \mathrm{Myr}$. The poorly studied HIP 28944 cluster (Platais et al. 1998) seems to lie in the continuation of the stream. Although uncertain, its age ( $\sim 60 \mathrm{Myr})$ fits the age sequence well, and it might be related to the stream.

Even at the relatively coarse scale of our KDE analysis, some sub-structures are apparent along the stream and call for a revision of the canonical division into a limited number of associations inherited from Kapteyn (1914) and Blaauw (1946).

Blaauw (1946) defined the Sco-Cen complex limits based on the spatial and velocity coherence of OB stars: beyond LCC, the proper motions and radial velocities of $\mathrm{OB}$ stars indeed become inconsistent, which was interpreted as a different origin. Nevertheless, Blaauw (1946) mentioned that his group 5 displayed a similar mean velocity as Sco-Cen members (made of his groups 2 to 4). Group 5 mostly consists of IC 2602 and IC 2391 members, but Blaauw (1946) did not succeed in conclusively associating them with the Sco-Cen complex. Blaauw probably missed the extension of Sco-Cen towards NGC 2451A and Collinder 135 because of the superposition of the Vela complex and the Galactic plane in the line of sight between IC 2391 and NGC 2451A. More recently, de Zeeuw et al. (1999) probably 


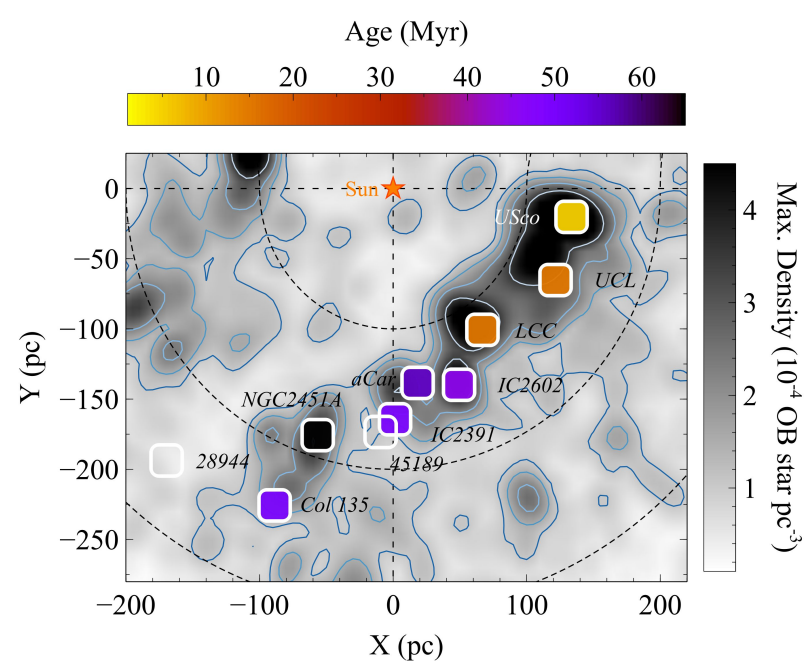

Fig. 2. Maximum density of OB stars projected on the galactic plane towards the Sco-CMa stream. The positions of known OB associations and clusters are indicated with squares. The colour scale is proportional to their age when an estimate is available in the literature and transparent otherwise. The location of the sun is indicated. This should be read jointly with Fig. 1.

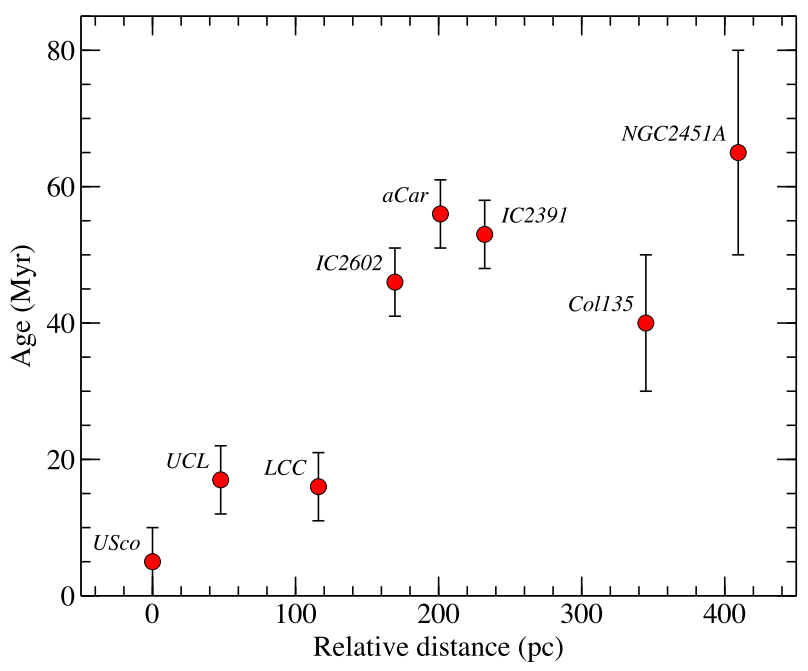

Fig. 3. Estimated age vs. distance from Upper Scorpius for the clusters and associations of the Sco-CMa stream.

also missed the connection because they were guided by the boundaries defined in Blaauw's earlier work. In that area, the Vela members clearly dominate and indeed have a different velocity distribution. The Sco-Cen or Sco-OB2 association should therefore probably be renamed the Sco-CMa OB association.

\subsection{Vela blue stream}

A second large complex is revealed around $l \sim 260^{\circ}$ and includes at least

- two OB associations: Vel OB2 and Trumpler 10, and

- four clusters: $\gamma$ Velorum, NGC 2547, NGC 2516, NGC 2451B.

A number of noticeable features are uncovered in the region. An as yet unknown but still rich and extended over-density clearly appears in front of $\gamma$ Velorum. The existence of a widespread
Table 2. Dispersion of age and distance measurements for the clusters and associations in Vela.

\begin{tabular}{lcccc}
\hline \hline Group & Distance & Ref. & Age & Ref. \\
\hline$\gamma$ Vel & $336-424$ & $(1),(2)$ & $3-5$ & $(3),(4)$ \\
NGC 2451B & $302-489$ & $(1),(5)$ & $44-170$ & $(1),(5)$ \\
NGC 2547 & $361-457$ & $(1),(2)$ & $20-78$ & $(2),(5)$ \\
Vela OB2 & $410-421$ & $(1),(7)$ & $15-20$ & $(1),(7)$ \\
Tr 10 & $366-424$ & $(1),(2)$ & $24-35$ & $(5),(7)$ \\
NGC 2516 & $373-409$ & $(1),(5)$ & $113-298$ & $(1),(5)$ \\
\hline
\end{tabular}

References. (1) Dias et al. (2002); (2) Anderson \& Francis (2012); (3) Eldridge (2009) ; (4) Jeffries et al. (2009); (5) Kharchenko et al. (2013), (6) Kharchenko et al. (2005); (7) de Zeeuw et al. (1999)

population of young stars in Vela has recently been suggested by the Gaia-ESO survey collaboration, who reported a new and distinct kinematic population in the line of sight to the $\gamma$ Velorum and NGC 2547 clusters (Jeffries et al. 2014; Sacco et al. 2015). They concluded that this population must be slightly younger and in front of the 5-10 Myr old $\gamma$ Velorum cluster. All evidence suggests that the extended population seen in our density map corresponds to the massive stars of this population, which encompasses a vast region of approximately $6^{\circ} \times 10^{\circ}$, on the sky and includes 24 OB stars of our sample (see Table 3). In the continuity of the current nomenclature, we hereafter refer to this group as the Vela OB5 association. The 3D spatial coincidence of Vela OB5 with the Vela supernova remnant (SNR263.9-3.3, see Fig. 1) suggests that its progenitor (the Vela pulsar, PSRB0833-45) must belong to it (Cha et al. 1999). We note that part of the Vela OB5 population could consist of some of the Vela OB2 and $\gamma$ Velorum members if their true distances were to turn out to be closer than the currently assumed values. It is unlikely, however, that all Vela OB5 candidate members are in this situation, and the density enhancement must be real.

Figure 5 shows a projection of the maximum density of OB stars on the galactic plane in the Vela region, together with the known clusters and $\mathrm{OB}$ associations found in that area of the Galaxy including the new Vela OB5 population ${ }^{1}$. At first glance, the coherence of the age distribution is not as obvious as for Sco-CMa. But except for NGC 2451B and NGC 2516, the stellar groups are all perfectly aligned with the main density enhancements and are ordered by increasing age from the new Vela OB5 (for which we assume an age of $\lesssim 5 \mathrm{Myr}$ as suggested by Jeffries et al. 2014) to the 35 Myr old NGC 2547 open cluster. NGC 2451B seems to lie relatively far away from the collimated stream defined by the main overdensity, and we infer that it does not belong to the Vela complex. We also note that its distance is still largely uncertain, ranging from 300 to $410 \mathrm{pc}$ (the former being used in Fig. 5). At this stage, its origin remains unclear. NGC 2516 can confidently be discarded as a member of the young Vela complex given its advanced age (Table 2), and we regard it as an unrelated galactic open cluster passing through on its orbit around the galactic centre.

The Vela stream apparent in our analysis covers $\sim 150 \mathrm{pc}$ and a period of $\sim 35 \mathrm{Myr}$ of star formation. This should be regarded as a lower limit, as the extension of the stream is set here by the sensitivity limit of Hipparcos. Gaia will hopefully reveal its full extension, which could reach 1 to $2 \mathrm{kpc}$ (Pettersson 2008), and

\footnotetext{
1 The positions of the known groups, computed using the de Zeeuw et al. (1999) positions, do not always match the position of density enhancements perfectly. We interpret the slight discrepancy as the result of the differences between the samples of members and the biases mentioned in Sect. 2 .
} 

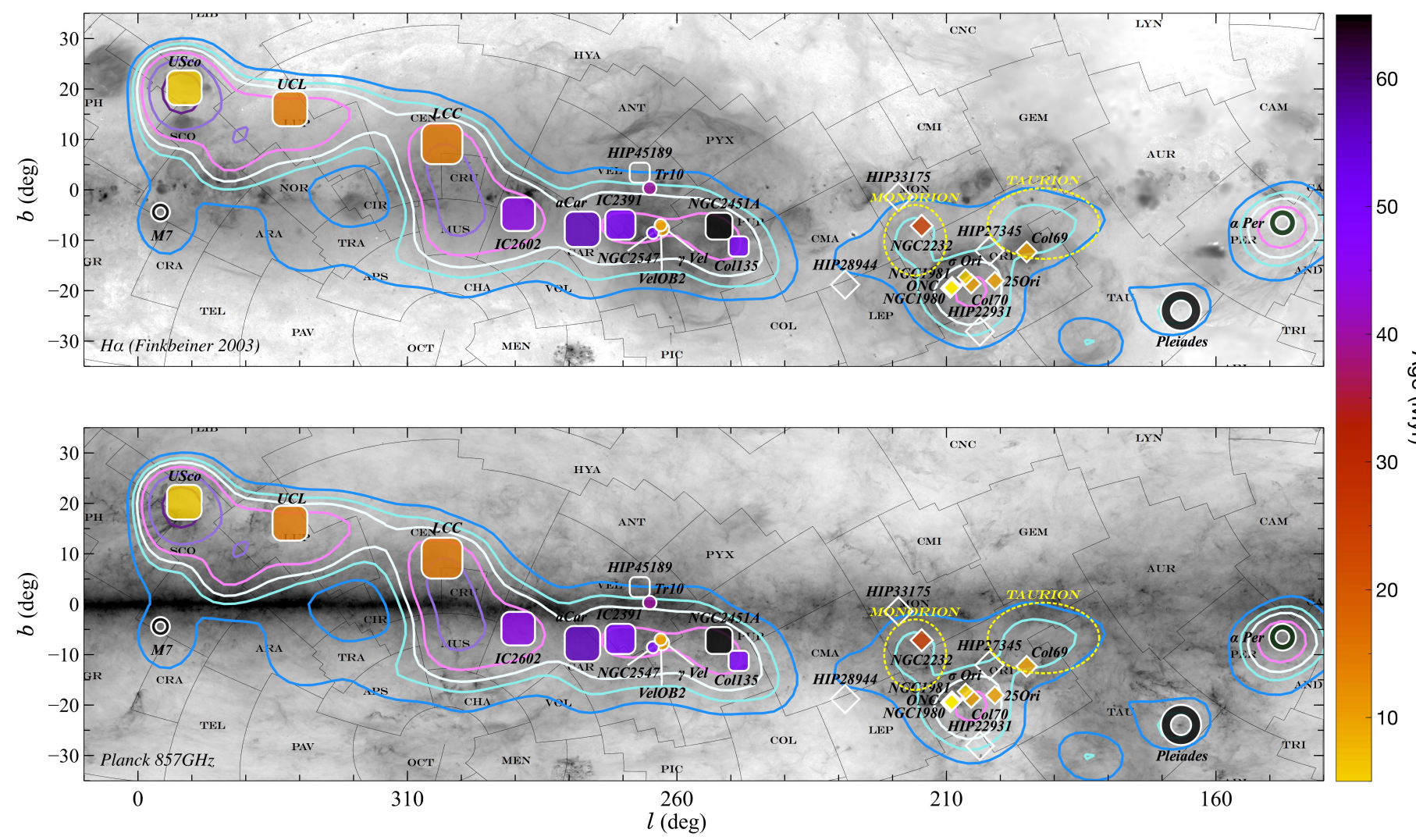

Fig. 4. Maximum density of OB stars projected on an H $\alpha$ (top panel, Finkbeiner 2003) and Planck $857 \mathrm{GHz}$ (lower panel Planck Collaboration I 2015) image of the sky. Clusters and associations belonging to the Sco-CMa stream are represented by squares. Clusters and associations in the Vela stream are represented by dots. Clusters and associations in the Orion blue stream are represented by diamonds. The Pleiades, $\alpha$-Per and Messier 7 are represented as circles. The symbol size is inversely proportional to the group distance. The colour scale is proportional to the age when an estimate is available in the literature and transparent otherwise. See also Fig. 1.

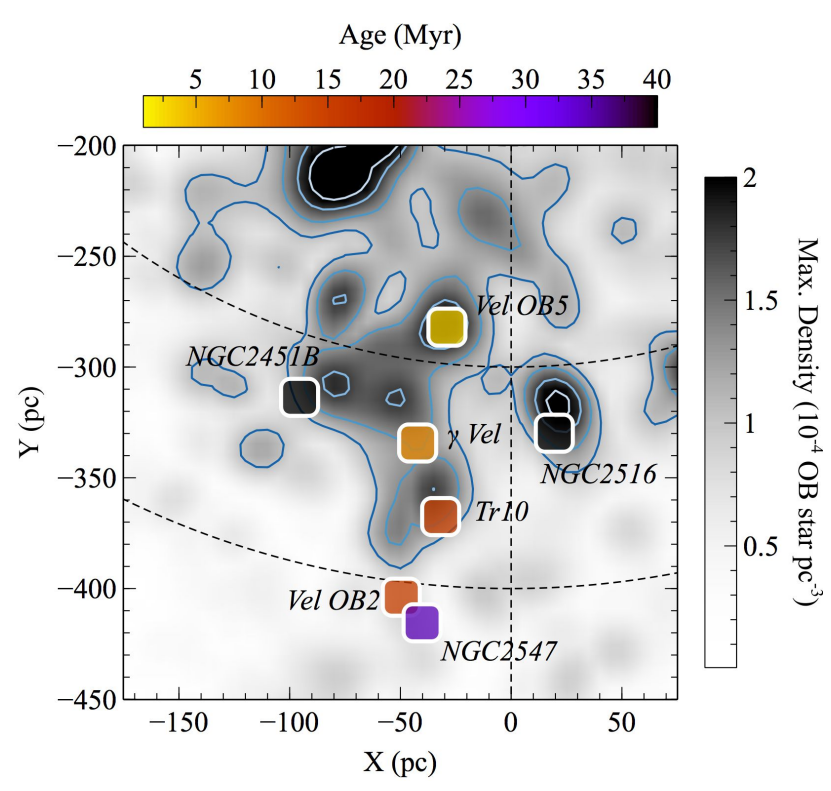

Fig. 5. Maximum density of $\mathrm{OB}$ stars projected on the galactic plane. The positions of Vel OB5, $\gamma$ Velorum cluster, Vel OB2, Tr 10, and NGC 2547 are indicated. The colour scale is proportional to their age when an estimate is available in the literature and transparent otherwise. This should be read jointly with Fig. 1 .

clarify the currently uncertain definition, distances, and ages of the young OB associations and clusters in Vela (Table 2).

\subsection{Orion blue stream: the forest in front of the tree}

The third prominent blue stream visible in the 3D density map is located towards $l \sim 200^{\circ}$ in the constellation of Orion. The iso-density surfaces represented in Fig. 1 extend from $150 \mathrm{pc}$ to at least $300 \mathrm{pc}$. A total of $48 \mathrm{OB}$ stars are included in the iso-surface, corresponding to $1 \times 10^{-4} \mathrm{OB}$ star $\mathrm{pc}^{-3}$ (silver isosurface in Fig. 1). They define a new group in Orion, at about $200 \mathrm{pc}$, and their complete member list is given in Table 3. This new group seems to include the two candidate associations centred on HIP 27345 and HIP 22931 reported by Platais et al. (1998) and the candidate cluster reported by Caballero \& Dinis (2008) centred on $\eta$ Ori. The well-known clusters and associations making the Orion OB1 complex (OB1a,b,c, and d, Blaauw 1964) are located at the far edge of this new group, and only Orion OB1a is above the detection threshold of the map. As already explained, the combination of their large heliocentric distance and line-of-sight extinction places Orion OB1abc below the iso-density levels represented in Fig. 1.

Figure 6 shows the well-known monotonic age sequence starting from the extremely young $\lesssim 1$ Myr clusters of Orion OB1d and sequentially passing through the $2 \sim 6 \mathrm{Myr}$ clusters of Orion OB1b and $\mathrm{c}$ and the $8 \sim 12 \mathrm{Myr}$ old Orion OB1a association (Bally 2008). The age of the new group is unknown, but the main-sequence lifetime of the giants and supergiants (of the order of $10 \mathrm{Myr}$ ) sets limits that are consistent with the proposed age sequence.

The front edge of the stream seems to start around $150 \mathrm{pc}$, but we speculate that Bellatrix ( $\gamma$ Ori), well aligned with the stream, 


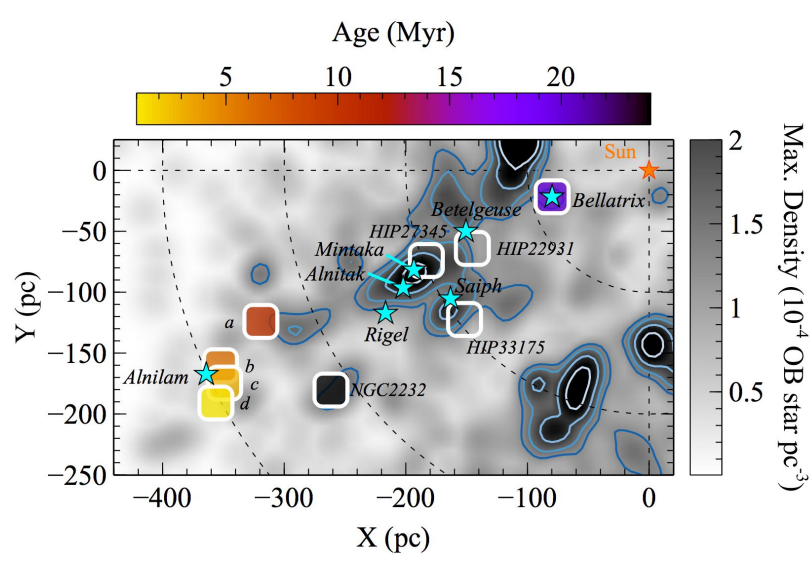

Fig. 6. Maximum density of $\mathrm{OB}$ stars projected on the galactic plane towards Orion. The positions of Orion OB1a,b,c, and d and Platais et al (1998) candidate associations and clusters are indicated with squares. The colour scale is proportional to their age when an estimate is available in the literature and transparent otherwise. The giants and supergiants composing the constellation asterism are represented by light blue stars. This should be read jointly with Fig. 1 .

could be part of it. Bellatrix is one of the supergiants that define the Orion constellation asterism. Located at a distance of $77 \pm$ $3 \mathrm{pc}$, its age is estimated to be $20_{-4}^{+2} \mathrm{Myr}$ (Janson et al. 2011) and is consistent with the age sequence of the stream. It coincides in space and age with the poorly studied $\sim 20$ Myr old 32 Ori cluster (Mamajek 2007), which should probably be called the Bellatrix cluster (see Alves \& Bouy, in prep.).

While there is abundant literature on the Orion OB1 groups, little information is available about the foreground population at $\sim 200 \mathrm{pc}$, even though it includes at least 48 OB stars. Platais et al. (1998) mentioned that the two candidate associations revealed by their analysis that are centred on HIP 27345 and HIP 22931 might be part of Orion OB1, a suggestion that unfortunately did not receive much attention in the literature. Our analysis suggests that these two groups and the $\eta$ Ori cluster candidate are related to a larger complex that is centred on the new group. The mostly radial motion of this group as seen from Earth placed it in an unfavourable configuration for searches that were based on the convergent point method (de Zeeuw et al. 1999). We note here as well that part of this new population could consist of some of the Orion OB1 groups if their true distances turn out to be closer than the currently assumed values.

As in the case of Sco OB2, the spatial coherence, age sequence, and the existence of a previously unknown rich group of B stars at about $200 \mathrm{pc}$ and in front of the well-known Orion OB1 complex calls for a revision of the sub-group boundaries and designations in that region. The currently adopted names and boundaries, Orion OB1,a,b,c, and d were defined according to their projected positions on the plane of the sky and do not reflect the 3D spatial coherence and age sequence. Until a standard naming convention of the Orion $\mathrm{OB}$ associations and clusters is adopted, we propose to refer to the new foreground population as Orion X.

The Orion stream is remarkably straight and collimated over more than $300 \mathrm{pc}$, suggesting a highly efficient linear propagation with little radial expansion for the corresponding OB associations (at least for their massive OB stars) over a timescale of $20 \sim 30$ Myr. The existence of a stream of B stars in Orion has been suggested almost a century ago by Charlier (1926), but has not been followed by the literature. In his plate IX, B stars of the Orion constellation clearly make a coherent and

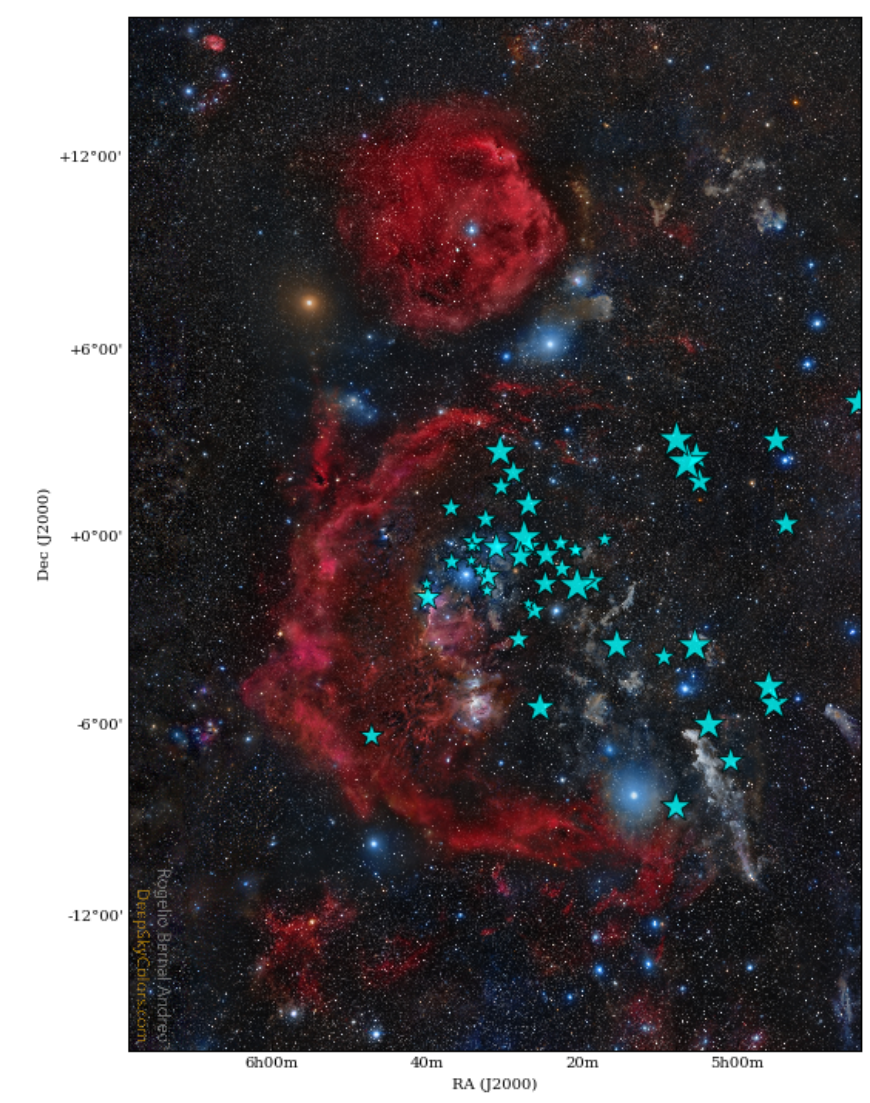

Fig. 7. OB star members of the new foreground group in the Orion stream. The symbol size is inversely proportional to the cube of the distance to enhance the perception of depth. Background photograph courtesy of Rogelio Bernal Andreo².

continuous structure dispersed along the line of sight from 80 to $600 \mathrm{pc}$. The proximity of the supergiants Saiph, Rigel, Bellatrix, Mintaka, and Alnitak ( $\kappa$ Ori, $\beta$ Ori, $\gamma$ Ori, $\delta$ Ori, and $\zeta$ Ori, respectively) has also been interpreted as circumstantial evidence for the existence of a young foreground population, but without making the connection to Charlier's stream of OB stars (Bally 2008). Bouy et al. (2014) recently reported the existence of a widely spread population located in front of and slightly older than Orion A. The existence of such a foreground population fits the present scheme perfectly, and its members are probably part of the Orion blue stream. As in the case of Vela, the superposition of all the associations and clusters of the Orion stream in the line of sight (Fig. 7) has led to great confusion regarding the distances and ages of the stellar groups belonging to the Orion OB1 complex (Genzel et al. 1981; Brown et al. 1994; Hirota et al. 2007; Sandstrom et al. 2007; Jeffries 2007; Lombardi et al. 2011; Alves \& Bouy 2012; Bouy et al. 2014). Given that it might be one of the richest OB associations closest to Earth, follow-up observations to find its members and characterize its properties are very much needed. A detailed study of the Orion stream will be presented in a future study.

The Orion stream is flanked by two major density enhancements that are almost superimposed on the plane of the sky near $l \sim 215^{\circ}$. The first one is found above and parallel to the Orion stream at an heliocentric distance of $\sim 200 \mathrm{pc}$. It seems to include (or coincide) with the candidate association centred on HIP 33175 reported by Platais et al. (1998). Because it is spread

http://www. deepskycolours.com 


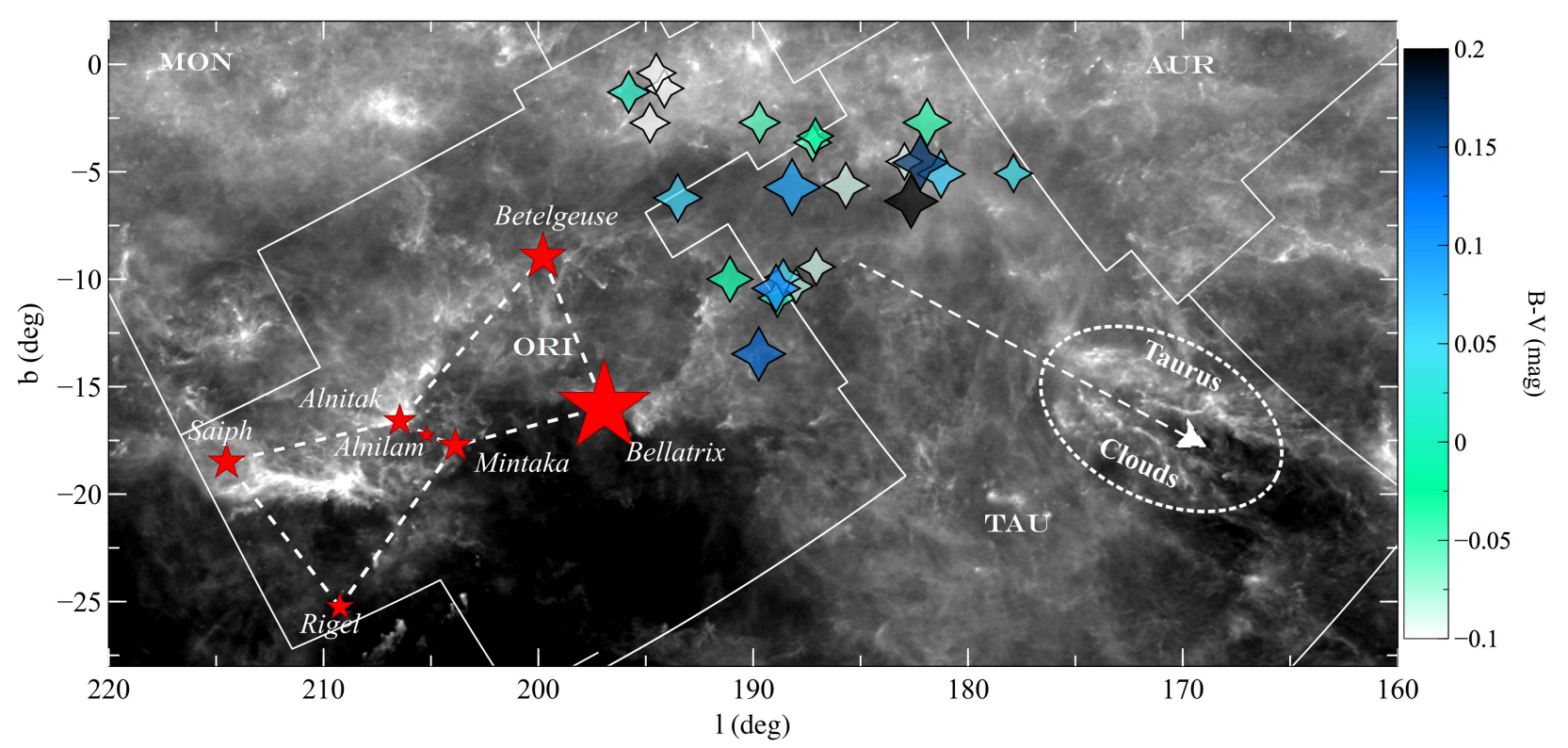

Fig. 8. Chart showing the B star members of the newly discovered Taurion association (stars). The colour scale represents their $(B-V)$ colour). The giants and supergiants composing the Orion constellation asterism are represented with red stars. The Taurus Molecular Clouds are indicated.

across the Orion and Monoceros constellations, we name this group the Monorion association. The members of this new candidate group are given in Table 3 . The second is found at an heliocentric distance of $\sim 320 \mathrm{pc}$ in the same direction, and it corresponds to the $30 \mathrm{Myr}$ old NGC 2232 cluster. These two young groups might be related to the Orion stream, but their nature and origin remain uncertain at this time. Follow-up velocity and age measurements are required to draw further conclusions and understand their possible relation with the Orion stream.

\subsubsection{Betelgeuse family and the Taurus molecular cloud}

Another previously unreported large and rich over-density of OB stars calls for attention. It is located between Orion and Taurus, and we hereafter refer to it as the Taurion OB association. It covers an area of $\sim 20^{\circ} \times 15^{\circ}$ and includes $36 \mathrm{~B}$ stars of our sample lying at an average distance of only $\sim 150 \mathrm{pc}$ from the Sun. Figure 1 shows that the position and velocity of Betelgeuse ( $\alpha$ Ori) suggests that it belongs to this new group and not to Orion, probably solving the long-standing puzzle of its origin (see Pettersson 2008, for a discussion). Taurion members are only 30 to $50 \mathrm{pc}$ away from the Taurus Molecular Cloud (TMC). Figure 8 shows that the TMC main filaments seem to be radially aligned with the B stars in Taurion, suggesting that stellar feedback (stellar winds and past supernovae) might be responsible for at least shaping the Taurus molecular cloud complex, but most likely more, possibly triggering star formation. Surprisingly, Taurus could therefore be an analogue of the Ophiuchus complex, where the feedback from Upper Scorpius OB stars has a dominant effect on the Ophiuchus complex by shaping filaments and triggering star formation (Alves et al., in prep.). The age of Taurion is uncertain, but the main-sequence lifetimes of their most massive members sets limits in the range between $\sim 10$ and $\sim 40$ Myr. Given that this might be one of the closest OB associations to Earth, follow-up work to characterize the Taurion association is urgently needed.

We find that seven Taurion members are included in the list of candidate members of the Cas-Tau OB association reported in de Zeeuw et al. (1999). Given the high expected contamination rate among their sample of Cas-Tau candidate members (between 58 and $70 \%$, as estimated by the authors themselves), we consider that Taurion members have very likely been mistaken as Cas-Tau members. We also note that the Taurion association does not coincide with the association of $16 \mathrm{~B}$ stars reported by Walter \& Boyd (1991), which are mostly centred on the TMC.

\section{Discussion}

\subsection{Solving long-standing puzzles}

Of the seven stars that compose the asterism of the Orion constellation, the distance of Alnilam ( $\epsilon$ Ori) is the only one that is consistent with the stellar groups composing Orion OB la,b,c, and $d$. The origin of the blue supergiants that define the body and belt of the ancient hero has been an open question for a long time. The proximity of Rigel, Saiph, Bellatrix, Alnitak, and Mintaka, all located between 80 and $250 \mathrm{pc}$, were considered incompatible with having a common origin with the Orion OB1 association. The discovery of the Orion stellar stream offers a simple solution to this puzzle, suggesting that these relatively distant populations are in fact genetically linked and part of a large galactic structure covering more than $300 \mathrm{pc}$ and at least 25 Myr of star formation history. The mystery of the Betelgeuse origin, the red giant marking the arm of the mythical hero (see Bally 2008), has been discussed in Sect. 3.3.1. Finally, the shape and extension of the stream provide a simple explanation for several aspects of the Orion Eridanus super-bubble such as its high elongation towards the Sun and the apparent proximity of its near wall, estimated to lie at a distance of $180 \mathrm{pc}$ (Diehl et al. 2004; Pon et al. 2014a,b). As recently suggested by Ochsendorf et al. (2015), the Orion-Eridanus bubble is probably the result of a sequence of shells superimposed along the line of sight. This view fits the results in this paper best, suggesting that the Orion-Eridanus bubble encloses the Orion stream, which is seen from Earth almost along the same line-of-sight. 


\subsection{Sequential vs. triggered star formation: from blue streams to $T$ - and $R$-associations}

The blue streams revealed by our analysis encompass 20 to $60 \mathrm{Myr}$ of star formation history. The continuous age sequence observed in each of them and their high level of collimation indicate a mostly linear star formation propagation. Interestingly, the blue streams are flanked by very young associations or remnant clouds. Sco-CMa is bordered by several T-associations: $\rho$-Oph ( 1 Myr), CrA (1 3 Myr), Lupus I-IV (1 3 Myr), Cha I-III (1 $\sim 3$ Myr), some loose low-mass clusters $\eta$ Cha (8 Myr) and $\epsilon$ Cha (6 Myr), the loose 40 Myr Argus moving group, and several starless remnant dark clouds: the Dark Horse or Pipe Nebulae, and the Musca and Lupus V and VI clouds. Similarly, Vela is surrounded by numerous cometary globules and the young Vela R1 association, while many cometary and dark clouds are associated with Orion.

This architecture reveals two types of star formation events: the primary and dominant mode creates the blue streams, large elongated galactic structures with a fairly well-defined age gradient, containing dense clusters and loose associations. A few Myr later, it is followed by a secondary event caused by the feedback from the stream's massive stars, which push and compress the surrounding interstellar medium, inducing star formation in lowmass clouds peripheral to the stream. This picture also provides an a posteriori justification for the choices made previously when selecting only stellar groups that follow a clear age sequence as stream components.

\subsection{Gould belt}

The definition and associated list of stellar groups of the Gould belt vary much from author to author. But because the main structure of the Gould belt is mainly determined by the Sco OB2 and Orion OB1 associations, there is a general good agreement regarding its geometry. Figure 1 shows the best-fit ellipse reported by Perrot \& Grenier (2003; shown in magenta). It appears that this model (or any other ellipse) gives a poor fit to the spatial distribution of OB stars revealed by our analysis, calling for a new interpretation of the distribution of stellar groups in the solar neighbourhood.

We here propose a revised description of the stellar solar neighbourhood that we argue consists of three major components: a) disk stars; b) old galactic clusters on their orbit around the galactic centre; and c) large-scale streams of young stars, which are approximately linear structures of a few to several hundred parsecs long, with a measurable age gradient. These blue streams seem to be the counterpart of similar stellar streams that are ubiquitous in nearby spiral galaxies (Elmegreen 2011). This revised view does not seem to support the existence of a ring-like structure such as the Gould belt. We argue that the Gould belt is then likely to be a projection effect produced by the Sun's vantage point between two large streams of young stars. The apparent combined rotation and expansion of the Gould belt could simply be due to the streams relative motions associated with galactic dynamics, but this would need to be modelled in detail.

\section{Conclusions}

We have studied the spatial density of OB stars within a box of $500 \times 500 \times 200 \mathrm{pc}$ in $X Y Z$ galactic coordinates centred on the Sun using the Hipparcos survey and a 12 pc wide kernel density estimator. The HipParcos survey is not complete over the volume covered by our study and is biased by extinction and multiplicity. The fixed KDE bandwidth also biased our analysis towards certain spatial scales and misses some known, more sparse, stellar structures in the solar neighbourhood. Within these limitations, we found the following results.

1. The 3D density map reveals three major elongated, streamlike structures containing several tens of OB stars, most of which are well-known clusters, and a number of previously unreported stellar groups.

2. The first structure runs from Scorpius to Canis Majoris and includes Upper Sco, UCL, LCC, IC 2602, IC 2391, a Car, Collinder 135, NGC 2451A, and possibly the HIP 28944 stellar group. It covers more than $350 \mathrm{pc}$ and at least $65 \mathrm{Myr}$ of star formation history.

3. The second structure is located in Vela and includes $\gamma$ Velorum, Vela OB2, Trumpler 10, NGC 2547, and a new group located at $\sim 200 \mathrm{pc}$ that we associate with the dispersed foreground population recently reported by Jeffries et al. (2014) and with the Vela supernova remnant SNR263.9-3.3. It covers at least $150 \mathrm{pc}$ and $30 \mathrm{Myr}$ of star formation history, but most likely extends well beyond the horizon of our study, which is set by the sensitivity of Hipparcos .

4. The third structure is located in the constellation of Orion, starts at a heliocentric distance of $\sim 150 \mathrm{pc}$, and extends to the well-known stellar groups included in the Orion OB1a,b,c, and $\mathrm{d}$ associations. It covers $300 \mathrm{pc}$ and at least $20 \mathrm{Myr}$ of star formation history. We speculate that it includes the 25 Myr old Bellatrix cluster, possibly placing the Orion complex as close as $80 \mathrm{pc}$ to the Sun.

5. A previously unreported stellar density enhancement is found between the Orion blue stream and Taurus molecular clouds, and we named it the Taurion OB association. Located at an heliocentric distance ranging between 130 and $170 \mathrm{pc}$, this $10 \sim 40 \mathrm{Myr}$ old group includes $36 \mathrm{~B}$ stars of our sample. Their stellar winds and past supernovae probably affected the Taurus molecular clouds that are located just $30 \sim 50$ pc away, and it might be responsible for the cloud structure and even triggering of star formation in this cloud complex.

6. Taurion is also the likely parent association of Betelgeuse, as suggested by the proximity of the red giant to the group and its velocity; this would solve the long-standing puzzle of the origin of Betelgeuse.

7. The architecture of the solar neighbourhood reveals two types of star formation events: the primary and dominant mode creates the blue streams, which are large galactic structures that are mostly linear and have an age gradient; they contain dense clusters and loose associations. In a secondary star formation event, the feedback from the massive members in the blue stream triggers star formation in peripheral low-mass clouds.

8. We find no evidence of a belt-like organization among the structures revealed by our analysis. We suggest that the Gould belt is a projection effect from the vantage point of the solar system between the newly identified stellar streams.

Acknowledgements. We are very grateful to the referee, Anthony Brown, for a constructive and thorough report that improved the manuscript substantially. We are also very grateful to Frédéric Arenou for enlightening explanations regarding the treatment of Hipparcos parallax measurement errors, and to Michail Vidiassov and David Barnes for their help with R, rgl and 3D PDF figures. We acknowledge the help of the anonymous specialists who provided their assistance on various StackExchange.com forums. H. Bouy is funded by the Ramón y Cajal fellowship program number RYC-2009-04497 and by Spanish Grant AYA201238897-C02-01. J. Alves acknowledges travel support from the ESAC Faculty 
council. This research has made use of the VizieR and Aladin images and cata$\log$ access tools and of the SIMBAD database, operated at the CDS, Strasbourg, France. This research has made use of Topcat (http://www.starlink.ac. uk/topcat/, Taylor 2005). This research made use of Astropy, a community developed core Python package for Astronomy (Astropy Collaboration et al. 2013). We acknowledge the use of the Legacy Archive for Microwave Background Data Analysis (LAMBDA), part of the High Energy Astrophysics Science Archive Center (HEASARC). HEASARC/LAMBDA is a service of the Astrophysics Science Division at the NASA Goddard Space Flight Center. Using observations obtained with Planck (http://www.esa.int/Planck), an ESA science mission with instruments and contributions directly funded by ESA Member States, NASA, and Canada.

\section{References}

Adler, D., Murdoch, D., \& others. 2015, rgl: 3D Visualization Using OpenGL, r package version 0.95.1247

Alves, J., \& Bouy, H. 2012, A\&A, 547, A97

Anderson, E., \& Francis, C. 2012, Astron. Lett., 38, 331

Astropy Collaboration, Robitaille, T. P., Tollerud, E. J., et al. 2013, A\&A, 558, A33

Bailer-Jones, C. A. L. 2015, PASP, in press [arXiv: 1507.02105]

Bally, J. 2008, Overview of the Orion Complex, ed. B. Reipurth, 459

Barnes, D. G., Vidiassov, M., Ruthensteiner, B., et al. 2013, PLoS ONE

Barrado y Navascués, D., Stauffer, J. R., \& Jayawardhana, R. 2004, ApJ, 614, 386

Blaauw, A. 1946, Ph.D. Thesis, Groningen University

Blaauw, A. 1964, ARA\&A, 2, 213

Bouy, H., Alves, J., Bertin, E., Sarro, L. M., \& Barrado, D. 2014, A\&A, 564, A29

Branham, Jr., R. L. 2002, ApJ, 570, 190

Brown, A. G. A., Arenou, F., van Leeuwen, F., Lindegren, L., \& Luri, X. 1997 in Hipparcos - Venice '97, eds. R. M. Bonnet, E. Høg, P. L. Bernacca, et al., ESA SP, 402, 63

Brown, A. G. A., de Geus, E. J., \& de Zeeuw, P. T. 1994, A\&A, 289, 101

Caballero, J. A., \& Dinis, L. 2008, Astron. Nachr., 329, 801

Cha, A. N., Sembach, K. R., \& Danks, A. C. 1999, ApJ, 515, L25

Charlier, C. V. L. 1926, Lund Medd

Claria, J. J., \& Kepler, S. O. 1980, PASP, 92, 501

de Bruijne, J. H. J. 1999, MNRAS, 306, 381

de Bruijne, J. H. J. 2000, Ph.D. Thesis, RA Leiden

de Zeeuw, P. T., Hoogerwerf, R., de Bruijne, J. H. J., Brown, A. G. A., \& Blaauw, A. 1999, AJ, 117, 354

Dias, W. S., Alessi, B. S., Moitinho, A., \& Lépine, J. R. D. 2002, A\&A, 389, 871

Diehl, R., Cerviño, M., Hartmann, D. H., \& Kretschmer, K. 2004, New Astron. Rev., 48, 81

Dobbie, P. D., Lodieu, N., \& Sharp, R. G. 2010, MNRAS, 409, 1002

Eddington, A. S. 1914, Stellar movements and the structure of the universe

Eggen, O. J. 1984, AJ, 89, 1350

Eldridge, J. J. 2009, MNRAS, 400, L20

Elias, F., Alfaro, E. J., \& Cabrera-Caño, J. 2006a, AJ, 132, 1052

Elias, F., Cabrera-Caño, J., \& Alfaro, E. J. 2006b, AJ, 131, 2700

Elmegreen, B. G. 2011, in EAS Pub. Ser. 51, eds. C. Charbonnel \& T. Montmerle, 31

ESA 1997, The HIPPARCOS and TYCHO catalogues. Astrometric and photometric star catalogues derived from the ESA HIPPARcos Space Astrometry Mission, ESA SP, 1200
Figueras, F., Gomez, A. E., Asiain, R., et al. 1997, in Hipparcos - Venice '97, eds. R. M. Bonnet, E. Høg, P. L. Bernacca, et al., ESA SP, 402, 519 Finkbeiner, D. P. 2003, ApJS, 146, 407

Galli, P. A. B., Teixeira, R., Ducourant, C., Bertout, C., \& Benevides-Soares, P. 2012, A\&A, 538, A23

Genzel, R., Reid, M. J., Moran, J. M., \& Downes, D. 1981, ApJ, 244, 884

Goodman, A. A. 2012, Astron. Nachr., 333, 505

Gould, B. G. 1879, Resultados del Observatorio Nacional Argentino, 1

Herczeg, G. J., \& Hillenbrand, L. A. 2015, ApJ, 808, 23

Herschel, Sir, J. F. W. 1847, Results of astronomical observations made during the years $1834,5,6,7,8$, at the Cape of Good Hope; being the completion of a telescopic survey of the whole surface of the visible heavens, commenced in 1825

Hirota, T., Bushimata, T., Choi, Y. K., et al. 2007, PASJ, 59, 897

Hoogerwerf, R. 2000, Ph.D. Thesis, RA Leiden

Hünsch, M., Weidner, C., \& Schmitt, J. H. M. M. 2003, A\&A, 402, 571

Janson, M., Bonavita, M., Klahr, H., et al. 2011, ApJ, 736, 89

Jeffries, R. D. 2007, MNRAS, 376, 1109

Jeffries, R. D., Naylor, T., Walter, F. M., Pozzo, M. P., \& Devey, C. R. 2009, MNRAS, 393, 538

Jeffries, R. D., Jackson, R. J., Cottaar, M., et al. 2014, A\&A, 563, A94

Kaltcheva, N. T., \& Hilditch, R. W. 2000, MNRAS, 312, 753

Kapteyn, J. C. 1914, ApJ, 40, 43

Kharchenko, N. V., Piskunov, A. E., Röser, S., Schilbach, E., \& Scholz, R.-D. 2005, A\&A, 438, 1163

Kharchenko, N. V., Piskunov, A. E., Schilbach, E., Röser, S., \& Scholz, R.-D. 2013, A\&A, 558, A53

Lada, C. J., Alves, J., \& Lada, E. A. 1996, AJ, 111, 1964

Lombardi, M., Alves, J., \& Lada, C. J. 2011, A\&A, 535, A16

Lutz, T. E., \& Kelker, D. H. 1973, PASP, 85, 573

Mamajek, E. E. 2007, in IAU Symp. 237, ed. B. G. Elmegreen \& J. Palous, 442

Ochsendorf, B. B., Brown, A. G. A., Bally, J., \& Tielens, A. G. G. M. 2015, ApJ, 808, 111

Pannekoek, A. 1929, Publ. Astron. Inst. University of Amsterdam, 2, 1

Pecaut, M. J., Mamajek, E. E., \& Bubar, E. J. 2012, ApJ, 746, 154

Perrot, C. A., \& Grenier, I. A. 2003, A\&A, 404, 519

Pettersson, B. 2008, Young Stars and Dust Clouds in Puppis and Vela, ed. B. Reipurth, 43

Planck Collaboration I. 2015, A\&A, submitted [arXiv: 1502 . 01582]

Platais, I., Kozhurina-Platais, V., \& van Leeuwen, F. 1998, AJ, 116, 2423

Plummer, H. C. 1913, MNRAS, 73, 492

Pon, A., Johnstone, D., Bally, J., \& Heiles, C. 2014a, MNRAS, 444, 3657

Pon, A., Johnstone, D., Bally, J., \& Heiles, C. 2014b, MNRAS, 441, 1095

R Core Team 2015, R: A Language and Environment for Statistical Computing, R Foundation for Statistical Computing, Vienna, Austria

Rasmuson, N. 1921, Lund Medd

Rosario, M., Tijerina, R. S., Cargile, P., \& James, D. 2013, in AASoc. Meet. Abstr., 221, 250.44

Sacco, G. G., Jeffries, R. D., Randich, S., et al. 2015, A\&A, 574, L7

Sandstrom, K. M., Peek, J. E. G., Bower, G. C., Bolatto, A. D., \& Plambeck, R. L. 2007, ApJ, 667, 1161

Stothers, R., \& Frogel, J. A. 1974, AJ, 79, 456

Subramaniam, A., \& Bhatt, H. C. 2000, Bull. Astron. Soc. India, 28, 255

Taylor, M. B. 2005, in Astronomical Data Analysis Software and Systems XIV, eds. P. Shopbell, M. Britton, \& R. Ebert, ASP Conf. Ser., 347, 29

Torres, C. A. O., Quast, G. R., da Silva, L., et al. 2006, A\&A, 460, 695

van Leeuwen, F. 2007, A\&A, 474, 653

Walter, F. M., \& Boyd, W. T. 1991, ApJ, 370, 318

Westin, T. N. G. 1985, A\&AS, 60, 99

Williams, P. M. 1967, Mon. Notes Astron. Soc. S. Afr., 26, 139 
H. Bouy and J. Alves: Cosmography of OB stars in the solar neighbourhood

Table 3. Members of the candidate new stellar groups.

\begin{tabular}{|c|c|c|c|c|c|}
\hline HIP & $l(\mathrm{deg})$ & $b(\mathrm{deg})$ & SpT & $\varpi$ (mas) & $\sigma_{\varpi}$ (mas) \\
\hline \multicolumn{6}{|c|}{ Monorion } \\
\hline 26304 & 211.01564 & -20.09731 & $\mathrm{~B} 8 \mathrm{~V}$ & 5.2 & 0.62 \\
\hline 26704 & 214.43576 & -20.43421 & B9 & 5.29 & 0.79 \\
\hline 27366 & 214.51434 & -18.49647 & B0Iab: & 5.04 & 0.22 \\
\hline 27402 & 213.34186 & -17.84582 & B9 & 5.48 & 0.91 \\
\hline 28278 & 216.81374 & -16.60746 & B9 & 5.17 & 0.66 \\
\hline 29401 & 212.58402 & -10.95355 & B9 & 5.68 & 0.69 \\
\hline 29497 & 216.43673 & -12.49364 & B9 & 5.23 & 0.59 \\
\hline 29629 & 212.82506 & -10.26840 & $\mathrm{~B} 5 \mathrm{~V}$ & 5.1 & 0.4 \\
\hline 31195 & 215.56882 & -6.59909 & B8 & 4.89 & 0.91 \\
\hline 30580 & 214.31767 & -7.93172 & B8 & 4.72 & 0.6 \\
\hline 30754 & 212.60434 & -6.48017 & $\mathrm{~B} 8 \mathrm{~V}$ & 5.05 & 0.81 \\
\hline 30843 & 213.71639 & -6.78918 & B9 & 4.67 & 0.85 \\
\hline 30852 & 216.59291 & -8.20861 & B9 & 5.38 & 0.74 \\
\hline 30867 & 216.66138 & -8.21395 & B4Veshell & 4.82 & 1.12 \\
\hline 31011 & 214.74008 & -6.80108 & B8V & 4.93 & 0.57 \\
\hline \multicolumn{6}{|c|}{ Taurion } \\
\hline 21881 & 176.64454 & -15.07176 & B3V & 8.19 & 0.88 \\
\hline 22013 & 176.90123 & -14.78227 & B9 & 8.49 & 0.81 \\
\hline 22910 & 172.49907 & -7.98077 & B9neqIV-V & 7.18 & 0.96 \\
\hline 23349 & 176.47400 & -9.45882 & B2IV & 5.83 & 1.23 \\
\hline 24789 & 189.74066 & -13.47409 & B9V & 8.57 & 1.03 \\
\hline 24984 & 177.87761 & -5.07662 & B9IV & 6.02 & 0.44 \\
\hline 25192 & 177.36331 & -4.09015 & B9Vs & 6.15 & 0.44 \\
\hline 25402 & 188.88025 & -10.88104 & B9V & 6.03 & 0.65 \\
\hline 25410 & 188.00455 & -10.31818 & $\mathrm{~B} 2 \mathrm{Vn}$ & 5.57 & 0.6 \\
\hline 25499 & 187.06574 & -9.42213 & $\mathrm{~B} 5 \mathrm{~V}$ & 5.94 & 0.34 \\
\hline 25555 & 188.93118 & -10.42417 & B9.5Vn & 7.69 & 0.33 \\
\hline 25572 & 182.64626 & -6.37402 & B9 & 8.56 & 1.09 \\
\hline 25657 & 188.59669 & -9.91077 & B9 & 5.8 & 0.69 \\
\hline 25695 & 181.25415 & -5.09909 & B9Vn & 7.67 & 0.73 \\
\hline 26062 & 182.21773 & -4.57608 & B8 & 8.78 & 0.61 \\
\hline 26093 & 191.08057 & -9.99400 & B3V & 7.31 & 0.38 \\
\hline 26248 & 182.95442 & -4.52068 & B2.5IV & 5.9 & 0.22 \\
\hline 26396 & 180.71933 & -2.65166 & B9IV-V & 6.46 & 1.01 \\
\hline 26451 & 185.68638 & -5.63604 & B2IVp & 7.33 & 0.82 \\
\hline 26640 & 181.89891 & -2.70865 & B3IV & 7.63 & 0.33 \\
\hline 26777 & 190.09292 & -7.31347 & B3IV & 5.13 & 0.81 \\
\hline 26925 & 188.18614 & -5.71967 & B9 & 9.06 & 0.54 \\
\hline 27364 & 193.17393 & -7.33029 & B2IV-V & 5.15 & 0.36 \\
\hline 27421 & 187.22969 & -3.64173 & B9Vn & 5.97 & 0.73 \\
\hline 27428 & 190.20447 & -5.37746 & B9 & 4.88 & 0.52 \\
\hline 27453 & 194.96568 & -8.06158 & B8 & 5.02 & 0.7 \\
\hline 27486 & 187.10118 & -3.33665 & B9 & 5.77 & 0.72 \\
\hline 27743 & 193.50911 & -6.21731 & B9spe... & 7.69 & 0.29 \\
\hline 28110 & 198.09006 & -7.65390 & B9Ib & 8.33 & 0.49 \\
\hline 28170 & 189.70110 & -2.70388 & B9 & 6.49 & 0.84 \\
\hline 28433 & 195.82974 & -5.31785 & B9 & 8.21 & 0.77 \\
\hline 29038 & 194.80645 & -2.72122 & B3V & 6.32 & 0.33 \\
\hline 29434 & 194.13443 & -1.11796 & $\mathrm{~B} 5 \mathrm{Vn}$ & 6.17 & 0.25 \\
\hline 29636 & 195.79703 & -1.29501 & B8V & 6.63 & 1.61 \\
\hline 29704 & 194.50990 & -.40248 & B7V & 6.24 & 0.68 \\
\hline 30120 & 202.49963 & -3.34023 & B9V & 6.15 & 1.03 \\
\hline
\end{tabular}


Table 3. continued.

\begin{tabular}{|c|c|c|c|c|c|}
\hline HIP & $l(\operatorname{deg})$ & $b(\mathrm{deg})$ & SpT & $\varpi$ (mas) & $\sigma_{\varpi}(\mathrm{mas})$ \\
\hline \multicolumn{6}{|c|}{ Orion $\mathrm{X}$ population } \\
\hline 22146 & 193.25795 & -25.29987 & B9 & 4.93 & 0.78 \\
\hline 22840 & 198.21778 & -25.45197 & B5V & 4.5 & 0.56 \\
\hline 22931 & 204.04145 & -28.01721 & B9 & 5.07 & 0.77 \\
\hline 22946 & 195.86351 & -23.75596 & B9 & 4.8 & 0.96 \\
\hline 22985 & 203.58915 & -27.61621 & B9 & 5.13 & 0.78 \\
\hline 23364 & 206.59365 & -27.68912 & B3V & 4.41 & 0.23 \\
\hline 23586 & 205.81569 & -26.53545 & B9 & 5.13 & 0.74 \\
\hline 23714 & 198.44898 & -22.36614 & $\mathrm{~B} 8 \mathrm{~V}$ & 4.57 & 1.11 \\
\hline 23745 & 203.56533 & -24.92205 & B8V & 5.32 & 0.76 \\
\hline 23757 & 197.75064 & -21.84711 & B8V & 4.77 & 0.8 \\
\hline 23843 & 198.10411 & -21.69334 & B8V & 5.76 & 0.87 \\
\hline 23916 & 208.95243 & -26.82634 & B8III & 4.79 & 0.47 \\
\hline 23957 & 197.59668 & -21.02031 & $\mathrm{~B} 8 \mathrm{~V}$ & 5.45 & 0.83 \\
\hline 24058 & 204.43332 & -24.22900 & B9V & 4.02 & 0.98 \\
\hline 24567 & 204.85571 & -22.71669 & $\mathrm{~B} 8 \mathrm{~V}$ & 5.09 & 1.14 \\
\hline 24709 & 201.82804 & -20.67830 & B5V & 3.43 & 0.8 \\
\hline 24847 & 203.31853 & -20.99823 & $\mathrm{~B} 1.5 \mathrm{Vn}$ & 4.56 & 0.73 \\
\hline 25025 & 203.68589 & -20.64855 & B8V & 5.47 & 0.6 \\
\hline 25028 & 202.63602 & -20.09369 & B3V & 3.25 & 0.37 \\
\hline 25044 & 202.63408 & -20.02653 & B2IV & 3.51 & 0.49 \\
\hline 25199 & 203.43083 & -19.95057 & B8V & 3.94 & 0.94 \\
\hline 25223 & 202.67607 & -19.49372 & $\mathrm{~B} 1.5 \mathrm{~V}$ & 3.72 & 0.33 \\
\hline 25365 & 203.26425 & -19.28132 & B9 & 4.65 & 0.58 \\
\hline 25368 & 204.15869 & -19.71384 & B3V & 4.44 & 0.47 \\
\hline 25401 & 208.02146 & -21.49108 & B9.5Vn & 4.9 & 0.36 \\
\hline 25480 & 205.14567 & -19.82146 & B2V & 4.19 & 0.61 \\
\hline 25548 & 203.27937 & -18.66151 & B9 & 4.33 & 0.7 \\
\hline 25557 & 205.04099 & -19.54900 & B5V... & 2.95 & 0.73 \\
\hline 25567 & 202.04422 & -17.96285 & B8V & 4.76 & 1.05 \\
\hline 25622 & 203.11053 & -18.37196 & B9 & 5.42 & 0.53 \\
\hline 25664 & 203.74395 & -18.57246 & B8 & 4.53 & 0.91 \\
\hline 25667 & 206.27886 & -19.83089 & B9V & 4.09 & 0.62 \\
\hline 25752 & 201.35272 & -17.01630 & B7V & 4.4 & 0.93 \\
\hline 25881 & 201.96558 & -16.89351 & B3V & 4.0 & 0.99 \\
\hline 25897 & 200.94873 & -16.29470 & B5V & 5.12 & 0.79 \\
\hline 25930 & 203.85592 & -17.73973 & $\mathrm{~B} 0 \mathrm{III}+\mathrm{O} 9 \mathrm{~V}$ & 4.71 & 0.58 \\
\hline 26012 & 204.85832 & -17.95496 & B9V & 4.5 & 0.95 \\
\hline 26020 & 205.30778 & -18.16594 & $\mathrm{~B} 4 \mathrm{Vn}$ & 3.17 & 0.72 \\
\hline 26048 & 203.18813 & -16.98581 & $\mathrm{~B} 8 \mathrm{~V}$ & 4.02 & 0.7 \\
\hline 26106 & 204.79282 & -17.63681 & $\mathrm{~B} 2.5 \mathrm{~V}$ & 3.22 & 0.37 \\
\hline 26188 & 204.06002 & -17.01074 & B5 & 4.33 & 0.74 \\
\hline 26210 & 204.22136 & -17.03396 & B7V & 3.01 & 0.67 \\
\hline 26213 & 204.65781 & -17.24181 & B3V & 2.95 & 0.57 \\
\hline 26464 & 205.01263 & -16.70000 & B6V & 3.72 & 0.72 \\
\hline 26481 & 203.41597 & -15.82245 & B5V & 3.93 & 0.77 \\
\hline 26727 & 206.45218 & -16.58516 & O9.7Ib+B0III & 4.43 & 0.64 \\
\hline 26742 & 206.07266 & -16.34199 & B2IV & 3.03 & 0.55 \\
\hline 27368 & 211.46337 & -17.07390 & B6V & 4.13 & 0.72 \\
\hline
\end{tabular}


H. Bouy and J. Alves: Cosmography of OB stars in the solar neighbourhood

Table 3. continued.

\begin{tabular}{cccccc}
\hline \hline HIP & $l(\mathrm{deg})$ & $b(\mathrm{deg})$ & SpT & $\varpi(\mathrm{mas})$ & $\sigma_{\varpi}(\mathrm{mas})$ \\
\hline \multicolumn{5}{c}{ Vela OB5 } \\
\hline 37966 & 265.38029 & -13.33378 & B8/B9II & 4.11 & 0.34 \\
38500 & 263.37989 & -11.19359 & B1.5Vp & 4.3 & 0.15 \\
38957 & 263.47560 & -10.27922 & B1Vp+B2: & 3.4 & 0.29 \\
39033 & 265.51143 & -11.25649 & B9II/III & 3.53 & 0.35 \\
39184 & 268.02213 & -12.36775 & B5Vn & 4.25 & 0.25 \\
39496 & 269.03418 & -12.34057 & B9.5V & 4.5 & 0.58 \\
39717 & 263.31515 & -8.48454 & B8V & 3.43 & 0.47 \\
39768 & 262.56447 & -7.90892 & B8IV & 3.7 & 0.47 \\
39773 & 264.07477 & -8.83058 & B8V & 4.5 & 0.31 \\
39919 & 263.92268 & -8.45434 & B8III & 3.56 & 0.25 \\
40077 & 263.91211 & -8.05289 & B3V & 3.87 & 0.27 \\
40152 & 266.97860 & -9.81185 & B9.5V & 4.14 & 0.4 \\
40594 & 263.14780 & -6.33849 & B9V & 4.52 & 0.54 \\
40742 & 263.57480 & -6.27456 & B8V & 3.45 & 0.4 \\
40749 & 264.41190 & -6.80631 & B3III & 3.47 & 0.32 \\
41053 & 265.45196 & -6.80155 & B8V & 3.91 & 0.4 \\
41223 & 270.91691 & -10.07899 & B6III/IV & 3.93 & 0.3 \\
41296 & 267.94257 & -7.93096 & B2Ve & 4.69 & 0.22 \\
41386 & 266.19294 & -6.52025 & B8V & 3.6 & 0.5 \\
41539 & 268.22234 & -7.60704 & B3IV & 3.48 & 0.46 \\
41897 & 271.36623 & -9.01478 & B9.5V & 3.9 & 0.36 \\
42121 & 270.83315 & -8.17669 & B7V & 4.12 & 0.33 \\
43499 & 274.99663 & -8.43088 & B8III & 4.42 & 0.2 \\
43763 & 275.75066 & -8.47458 & B5V & 4.58 & 0.34 \\
\hline
\end{tabular}

